\title{
Iron Pill Gastritis: An Under Diagnosed Condition With Potentially Serious Outcomes
}

\author{
Tagore Sunkara ${ }^{a}$, , Megan E. Caughey ${ }^{\mathrm{b}}$, Sofia Nigar ${ }^{\mathrm{a}}$, Raquel Olivo ${ }^{\mathrm{c}}$, \\ Vinaya Gaduputic
}

\begin{abstract}
Given the ubiquitous use of oral iron therapy, their side effects are often encountered and well recognized in clinical practice. However, iron pill gastritis remains an often under-reported and elusive diagnosis. An astute clinician should be aware of this condition in order to promptly discontinue oral iron and institute timely treatment. Here in, we present a case of a 46-year-old woman who presented to the gastroenterology clinic with vague epigastric pain and microcytic anemia. Esophagogastroduodenoscopy revealed multiple gastric erosions and non-bleeding gastric antral ulcer with biopsies showing excessive iron deposition suggestive of iron pill gastritis. We reviewed the clinical features, pathology, and treatment of iron pill gastritis along with the review of the literature.
\end{abstract}

Keywords: Iron pill gastritis; Iron sulfate; Ferrous sulfate; Gastritis; Gastric ulcer; Oral iron supplementation; Anemia

\section{Introduction}

There has long been a clinical association between mucosal injury of the upper gastrointestinal tract and conditions that involve an excess of iron accumulation, like hemochromatosis, cirrhosis, and multiple blood transfusions. In these conditions, the extra iron collects in the glands and lamina propria, producing hemosiderin deposits, which damage the gastric and duodenal mucosa [1]. It therefore makes sense that patients being treated for iron-deficiency anemia with oral iron supplementation would also be at risk for gastritis. Yet, the role

Manuscript accepted for publication February 20, 2017

a Department of Gastroenterology and Hepatology, The Brooklyn Hospital Center, Clinical Affiliate of The Mount Sinai Hospital, 121 Dekalb Avenue, Brooklyn, NY 11201, USA

${ }^{b}$ New York Institute of Technology College of Osteopathic Medicine, Old Westbury, NY, USA

'Department of Gastroenterology and Hepatology, SBH Health System, 4422 Third Avenue, Bronx, NY 10457, USA

${ }^{\mathrm{d} C}$ Corresponding Author: Tagore Sunkara, Department of Gastroenterology and Hepatology, The Brooklyn Hospital Center, Clinical Affiliate of The Mount Sinai Hospital, 121 Dekalb Avenue, Brooklyn, NY 11201, USA.

Email: tagoresunkara@hotmail.com

doi: https://doi.org/10.14740/gr804w of iron supplementation in the context of gastritis has not been extensively studied.

\section{Case Report}

A 46-year-old woman with co-morbidities of hypertension and iron deficiency anemia (on oral iron supplementation) presented to the gastroenterology clinic with vague epigastric pain, intermittent dark colored stools, and persistent anemia. Physical examination was unremarkable barring conjunctival pallor. The fecal occult blood test was positive. The patient was found to have microcytic, hypochromic anemia with a hemoglobin level of $7.1 \mathrm{~g} / \mathrm{dL}$. A diagnostic esophagogastroduodenoscopy revealed multiple gastric erosions and a non-bleeding gastric antral ulcer (Figs. 1 and 2). Biopsy of the ulcer revealed focal acute chronic gastritis with an aggregate of iron-rich material on the epithelial surface that was stained with hematoxylin and eosin (H\&E) and Prussian blue iron stain (Figs. 3 and 4). The patient was started on proton pump inhibitors and switched to intravenous iron therapy with resolution of her symptoms and correction of the anemia.

\section{Discussion}

Iron-deficiency anemia is typically treated with oral iron sup-

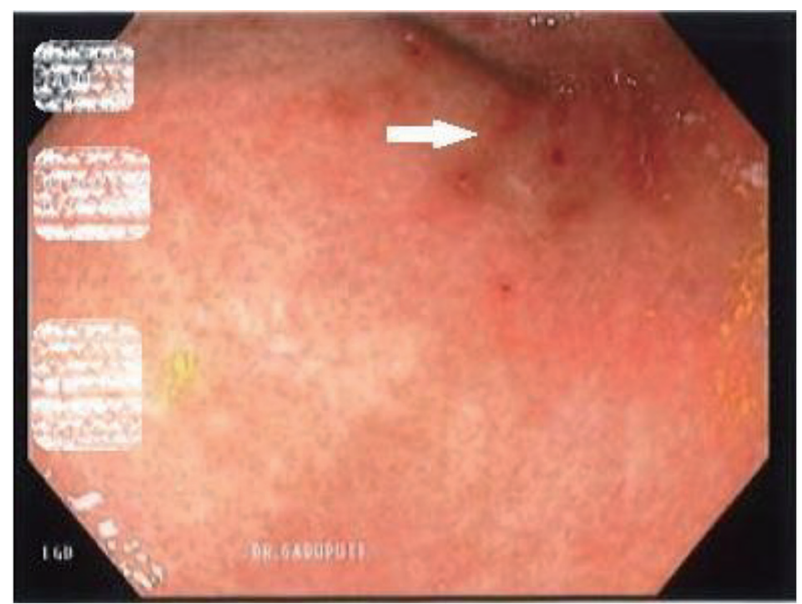

Figure 1. Esophagogastroduodenoscopy revealing non-bleeding clean based ulcers (white arrows). 


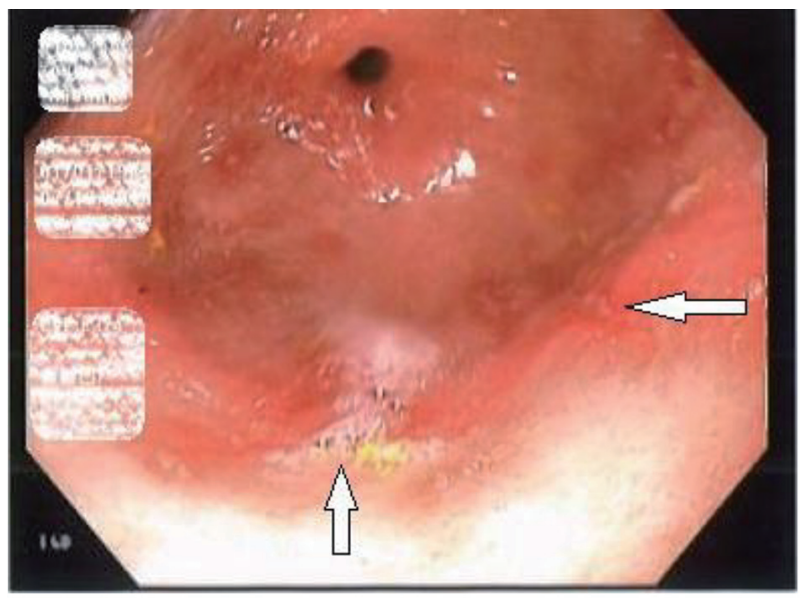

Figure 2. Esophagogastroduodenoscopy revealing erosions in the antrum of the stomach (white arrows).

plementation. The adverse outcomes that can result from an iron overdose are well documented in the literature. The complications of a standard therapeutic oral dose, however, have been less studied. One serious potential complication of iron supplementation is iron pill-induced gastritis. The proposed mechanism for how oral ferrous sulfate produces this gastritis involves iron oxidation and subsequent damage to the esophagus and stomach. More specifically, when iron is oxidized from the ferrous to ferric form, an epithelial injury occurs [2].

A study of 1,300 upper gastrointestinal tract biopsies from 33 patients identified crystalline iron deposits in $0.9 \%$ (or 12/1,300 cases total). Medication-associated damage was prevalent in about $0.7 \%$ (or $9 / 1,300$ cases total). This study first concluded that therapeutic iron ingestion both induces and exacerbates erosive mucosal injury. In particular, histology specimens revealed areas of mucosal ulceration overlapping with those of extracellular crystalline iron particle deposition. This observation was especially pronounced in patients with associated disorders of the esophagus and stomach [3].

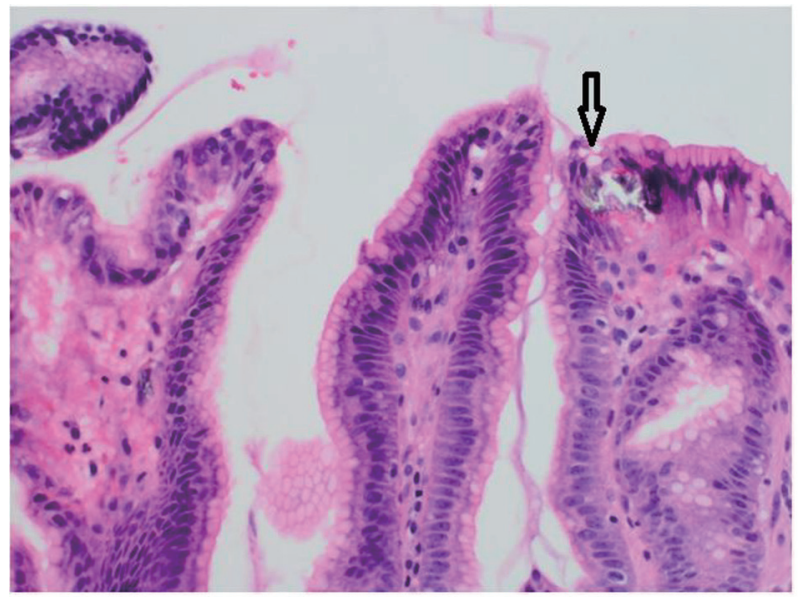

Figure 3. Biopsy of the gastric ulcer revealing focal acute chronic gastritis with an aggregate of iron-rich material on the epithelial surface (black arrow) that was stained with H\&E stain.

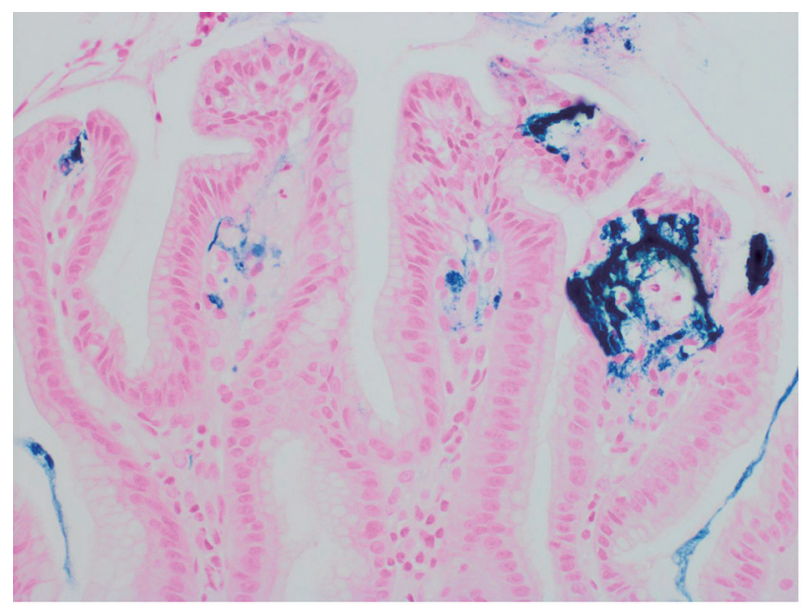

Figure 4. Biopsy of the gastric ulcer revealing focal acute chronic gastritis with an aggregate of iron-rich material on the epithelial surface that was stained with Prussian blue iron $(\times 400)$.

A case study of a 59-year-old male with iron deficiency anemia who was prescribed ferrous sulfate tablets serves as further evidence of this pathological phenomenon. This patient had co-morbidities of Child-Pugh B cirrhosis and esophageal varices, and on endoscopy, was found to have a 6-mm gastric ulcer in the gastric body. Biopsy results determined through H\&E staining that this ulcer was due to erosive gastritis, and an iron stain showed heavy iron deposition localized to the region of the ulcer. This patient was ultimately switched from oral iron tablets to a liquid iron supplement to prevent worsening of the iron-induced mucosal injury [4].

A liquid form of iron supplement is much less toxic to the gastric mucosa than solid iron tablets are. A liquid formulation does not produce the same epithelial injury as the iron tablet does because the liquid formulation is unable to concentrate within the body to the same extent. This is why in animal studies, even after consuming a lethal serum level of a liquid iron supplement, these same animals do not exhibit any signs of mucosal erosion [5].

The findings of a second study by Haig and Driman, involving 16 gastric, four duodenal, and five esophageal biopsies, are consistent with what was observed in the patient we are presenting. This study noted though that the average age of patients with erosive damage was $43 \%$ higher (76 versus 53 years of age, $\mathrm{P}=0.002$ ) in the group that was overloaded with iron compared to the group that was not. One epidemiological explanation for this would be that elderly patients are at greater risk for polypharmacy, gastric motility issues, and impediments to proper medication administration. Consequently, they are more likely to be taking iron pills, and by extension, are also more likely to develop supplemental iron-induced gastritis [1].

When examining the role of iron pills in upper gastrointestinal pathology, however, Kaye et al did not observe any significant associations with regard to age, gender, NSAID, or aspirin use. This retrospective study identified 59 patients, totaling 64 episodes of iron deposition. When further broken down, $86 \%$ $(6 / 7)$ of patients with esophageal iron deposition displayed ero- 
sion, compared to the $63 \%$ (29/46) of patients with gastric iron deposition who had erosion and the $80 \%$ of patients $(37 / 46)$ who had reactive gastritis. Perhaps most compelling of all though, this study determined that $98 \%$ of patients with iron deposition had a history of oral iron supplementation [6].

Given the common nature of iron deficiency anemia, the fact that iron pills have been shown to damage the mucosa of the upper gastrointestinal tract should not be taken lightly. Case studies documenting iron deposition injury to other anatomical regions, like the hypopharynx, have now also emerged [7]. Collectively, these studies are testament the systemic quality of the concern regarding oral iron supplements. On a positive note, liquid iron does not produce the same adverse effect of mucosal ulceration. It is a promising alternative therapy for those with iron deficiency anemia who are also suffering from iron-induced gastritis or erosion of the mucosa.

\section{Author Contributions}

Conception and design: Tagore Sunkara and Vinaya Gaduputi. Drafting of the article: Tagore Sunkara and Vinaya Gaduputi. Critical revision of the article for important intellectual content: Tagore Sunkara, Megan E. Caughey, Sofia Nigar, Raquel Olivo, and Vinaya Gaduputi. Final approval of the article: Tagore Sunkara, Megan E. Caughey, Sofia Nigar, Raquel Olivo, and Vinaya Gaduputi.

\section{References}

1. Haig A, Driman DK. Iron-induced mucosal injury to the upper gastrointestinal tract. Histopathology. 2006;48(7):808-812.

2. Aruoma OI, Halliwell B. Superoxide-dependent and ascorbate-dependent formation of hydroxyl radicals from hydrogen peroxide in the presence of iron. Are lactoferrin and transferrin promoters of hydroxyl-radical generation? Biochem J. 1987;241(1):273-278.

3. Abraham SC, Yardley JH, Wu TT. Erosive injury to the upper gastrointestinal tract in patients receiving iron medication: an underrecognized entity. Am J Surg Pathol. 1999;23(10):1241-1247.

4. Hashash JG, Proksell S, Kuan SF, Behari J. Iron Pill-Induced Gastritis. ACG Case Rep J. 2013;1(1):13-15.

5. Kothadia JP, Arju R, Kaminski M, Mahmud A, Chow J, Giashuddin S. Gastric siderosis: An under-recognized and rare clinical entity. SAGE Open Med. 2016;4:2050312116632109.

6. Kaye P, Abdulla K, Wood J, James P, Foley S, Ragunath $\mathrm{K}$, Atherton J. Iron-induced mucosal pathology of the upper gastrointestinal tract: a common finding in patients on oral iron therapy. Histopathology. 2008;53(3):311-317.

7. Cimino-Mathews A, Broman JH, Westra WH, Illei PB. Iron pill-induced tumefactive mucosal injury of the hypopharynx. Am J Surg Pathol. 2010;34(11):1720-1722. 